Fecha de recepción: mayo 2014 Fecha de aceptación: mayo 2015 Versión final: julio 2016

\section{Residuos agro-industriales utilizados \\ como materias-primas en estudios de desarrollo de fibras textiles}

Sirlene M. Costa ${ }^{\star}$ Esteban F. Tuesta ${ }^{\star *}$

$\&$ Silgia A. Costa ${ }^{* * *}$

Resumen: Los negocios agropecuarios en Brasil siempre ocuparon un lugar destacado en la economía y en el desarrollo del país. Actualmente, la mayor parte de los residuos de la caña de azúcar se queman en las centrales de producción de energía. Estos residuos se componen principalmente de celulosa, lignina y poliosas. En este artículo estos componentes fueron utilizados para producir fibras textiles. Para extraer la celulosa se llevó a cabo el despulpado alcalino y blanqueo con peróxido de hidrógeno. Las fibras obtenidas eran compatibles con Lyocell.

Palabras clave: bagazo de caña de azúcar - celulosa - fibras paja - Lyocell - textiles.

[Resúmenes en inglés y portugués en la página 309]

${ }^{(*)}$ Graduada en Ingeniería Industrial Química por la Escuela de Ingeniería de Lorena EELUSP (1996), Maestría en Biotecnología Industrial (1999) y Doctorado en Biotecnología Industrial (2005), ambos por la misma universidad. Post-doctorado por la Facultad de Ciencias Farmacéuticas - USP. Docente de la EACH / USP desde 2011 y orientadora en el programa de post-graduación del Curso de Textil y Moda.

${ }^{(* *)}$ Doctor en Ingenieria Eléctrica por la Escola Politécnica de la Universidade de São Paulo (Brasil) y Pós-Doctorado por el Laboratório de Estudios Métricos (LEMI) de la Universidad Carlos III de Madrid (España). Bachiller en Estadística por la Universidad Nacional Federico Villarreal, Lima (Perú). Master en Estadística por el Instituto de Matemática e Estatística (IME) de la Universidade de São Paulo (Brasil). Profesor en la Escola de Artes, Ciências e Humanidades de la Universidade de São Paulo desde 2005.

${ }^{(* *)}$ Profesora Asociada EACH/USP. Graduada en Ingeniería Industrial Química por la Escuela de Ingeniería de Lorena EEL/USP (1995). Maestría en Biotecnología Industrial por la Escuela de Ingeniería de Lorena EEL/USP (1998). Doctorado en Ingeniería Textil por la Universidad de Minho, Portugal (2002). Post-doctorado en Biomateriales. 


\section{Introducción}

En la actualidad existe una gran preocupación con los efectos causados por el impacto humano en el planeta y los asuntos relacionados con la dependencia de los productos derivados del petróleo extraídos de recursos naturales, cada vez más escasos y con costos crecientes. Hay una clara tendencia a revertir la matriz energética global dependiente del petróleo (Programa para la Agricultura y Medio Ambiente WWF - Brasil, 2008).

En la industria textil, la mayor parte de la producción de las fibras se obtienen a partir de materias primas naturales o sintéticos a partir de derivados del petróleo.

Las tendencias mundiales para el avance científico y tecnológico en el área de nuevos materiales destacan la importancia de la utilización de residuos industriales y agroindustriales como materia prima en los procesos de producción. La reutilización y el reciclaje de estos residuos pueden minimizar los problemas ambientales relacionados con la acumulación y disminuir el uso de materiales nobles (Machado, 2000, Pandey et al., 2000).

Brasil es el mayor productor mundial de caña de azúcar. La Companhia Nacional de Abastecimento - CONAB, divulgó una safra 2011/2012, mas de 624 millones de toneladas, siendo esa producción utilizada para la obtención del alcohol y azúcar. Lo que generó cerca de 208 millones de toneladas de bagazo de caña (CONAB, 2012). Los costos de los procesos de cosecha y lavado del bagazo están incluidos en el proceso de extracción del azúcar, volviendo de esta forma las condiciones económicas buenas para el procesamiento de este resíduo.

Además, la expectativa es que la cosecha da caña sea integralmente mecanizada hasta 2017 y que la paja no sea mas quemada en el campo, transformándose también en otro resíduo interesante para ser utilizado como materia-prima (UNICA, 2008). Por estos factores, la disponibilidad y composición del bagazo y la paja de la caña han impulsado investigaciones para el desarrollo de tecnologías de modo a aprovechar ese residuo de forma mas racional. El bagazo es un resíduo rico en carbohidrato siendo constituído por fibras y medula, en las proporciones de aproximadamente $65 \%$ y $35 \%$ respectivamente (Triana et al., 1990). Como todo material lignocelulósico el bagazo y la paja son constituídos por tres principales componentes macromoleculares: celulosa, poliosas y lignina.

La celulosa es un polímero lineal (parte amorfa y parte cristalina) formado por moléculas de anhidroglicosa unidas a traves de vínculos b-1,4 glicosídicas, de fórmula general $\left(\mathrm{C}_{6} \mathrm{H}_{10} \mathrm{O}_{5}\right)$ n. Este polímero esta compuesto por unidades monoméricas de celobiosa que se repiten presentando siempre el oxígeno que liga los anillos glicosídicos en la posição ecuatorial (Ferraz, 2001). Pudiendo ser modificado y convertido en derivados como los acetatos, éter y ésteres de celulosa (Fengel y Wegener, 1989).

Las poliosas (o hemicelulosas) son compuestas por los azúcares glicosa, manosa y galactosa (hexosas) y xilosa y arabinosa (pentosas), pudiendo aún presentar cantidades variables de ácidos uronicos y desoxiexoses en algunos tipos de vegetales. Las poliosas se presentan en la forma de polímeros ramificados de peso molecular menor que la celulosa y pueden ser homopolímeros (ejemplo: xilano, formado por xilosa) o heteropolímeros (ejemplo: glicomanana formado por glicosa y manosa). La cantidad de poliosa en diferentes tipos de vegetal es bastante variable, con un valor medio de 20\% (Fengel y Wegener, 1989). 
La lignina después de la celulosa es el compuesto orgánico mas abundante entre los materiales lignocelulósicos. Se compone basicamente de unidades de fenilpropano formando una macromolécula tridimensional y amorfa, representando cerca del 20 a 30\% del total de la madera. La lignina ejerce una función estructural en el complejo celular de la pared de plantas superiores, actuando como un pegamento que da cohesión al conjunto de células. La cantidad de lignina varia entre las diferentes especies de plantas superiores y también entre plantas de la misma especie (Fengel y Wegener, 1989).

En los estudios de Costa et al., 2013 ab y 2008, los autores utilizaron la celulosa del bagazo y de la paja de caña de azúcar para la obtención de una fibra artificial denominada Lyocell. Las fibras de Lyocell son el resultado de intensiva investigación en la fabricación de fibras naturales de origen celulósica de modo diferente al tradicional. El proceso de fabricación de la fibra de Lyocell se basa en un proceso de hilado en disolvente orgánico, utilizando para tal el NMMO (óxido de N-metilmorfolina).

En este proceso, el disolvente NMMO se recupera casi por completo (99,7\%), reciclado y enviado nuevamente para el proceso y el agua que es recuperada durante el proceso puede ser usada en las etapas de lavada posteriores de las fibras. O Lyocell es una fibra celulósica de última generación, considerada como la mas importante innovación en los últimos años dentro de la indústria de fibras textiles al nivel de aplicación en prendas de vestir convencional. Una de las principales características de la fibra de Lyocell es el toque suave, la alta capacidad de absorción lo que permite la producción de artículos confortables y fáciles de ser lavados (Rosenau et al., 2003; Fink et al., 2001). Además de la producción del Lyocell derivado del bagazo y de la paja, estudios fueron realizados con resíduos derivados de la indústria de la pesca el quitosano para obtención de una fibra hibrida con propriedades bactericida, fungicida y cicatrizante.

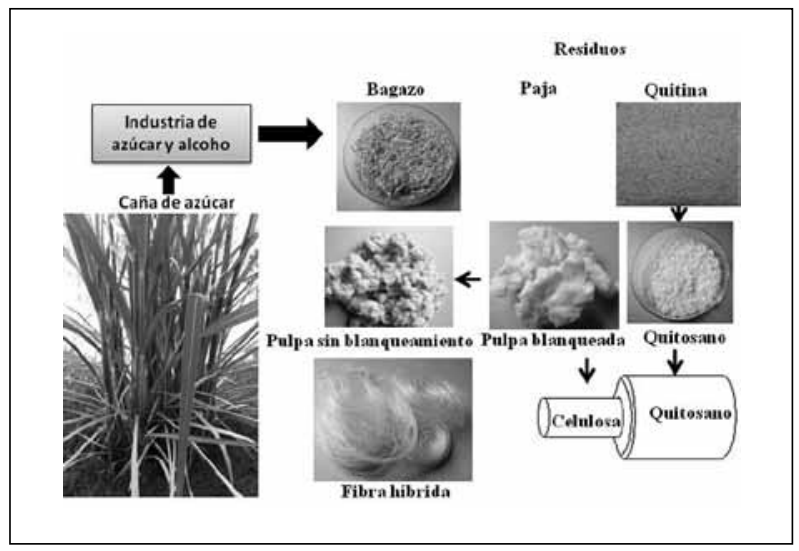

Figura 1. Fluxograma del proceso de producción de celulosa de bagazo y paja y fibras híbridas. Fuente. Elaboración propia. 
Todos los años, aproximadamente 100 mil millones de toneladas de quitina son producidas en el mundo, siendo esta cantidad comparada con la producción de celulosa por plantas de grande porte (Kong, 2010). La quitina es un polímero natural, siendo el segundo polisacarido mas abundante en la natureza después de la celulosa (Yamamoto et al., 2005, Alonso et al., 2010). Puede ser encontrada en abundancia em el exoesqueleto de la mayoria de los crustáceos como: cangrejos, camarones, langostas (Hoven et al., 2007) y cutículas de algunos insectos (Alonso et al., 2010). Los caparazones de los cangrejos, camarones, etc., provenientes de restos del procesamiento de comida (desechos de la industria pesquera) son actualmente la principal fuente de quitina (Hoven et al., 2007; Kong, 2010). La quitina es un polímero no ramificado formado por unidades de $\mathrm{N}$-acetil-D-glucosaminas, unidas por vínculos b-( $\left.{ }^{\circledR 4}\right)$, formando cadeias de varias centenas de unidades (Canella et al., 2001; Hoven et al., 2007). Su estructura química es semejante a la celulosa, en la cual los grupos hidroxila (celulosa) de la posición C-2, son substituídos pelo grupo acetamida (Pillai, et al, 2009).

La quitina puede ser encontrada en dos posibles organizaciones moleculares, la a-quitina (cangrejos, camarones, langostas) de orientación anti-paralela y la b-quitina (calamares y pulpos) de orientación paralela (Tolaimate et al., 2000). La formación a há sido la mas utilizada en aplicaciones médicas, a pesar que recientemente la b-quitina haya sido considerada como una potencial e importante fuente de quitina. La quitina es un polímero biodegradable, no tóxico y posee una elevada masa molar. Este polissacarido es insoluble en agua y en la mayor parte de los disolventes orgánicos (semejante a la celulosa en relación a la solubilidad y baja reactividad química), lo que vuelve su uso limitado en aplicaciones industriales (Tolaimate et al., 2000).

La quitina puede ser quimicamente modificada de modo a obtener otros polímeros derivados, como el quitosano, el sulfato de quitosano, el hidrocloreto de D-glicosamina, la carboximetilquitosano, el sulfato de glicosamina, entre otros. El quitosano es uno de los derivados de la quitina que puede ser solubilizado en soluciones de ácido acético diluído. Este polímero há sido ampliamente aplicado en los diversos campos de las ciencias médicas para el desarrollo de nuevos materiales con propiedades terapéuticas (Jayaumar, et al., 2011).

De las principales ventajas ofrecidas por polímeros naturales podemos destacar los hechos de que son de facil obtención, biocompatíbles y biodegradables. Tales ventajas son responsables por las crescientes investigaciones para posibles aplicaciones de esos polímeros en diversas areas, contribuyendo de forma significativa para la comunidad científica. La celulosa es un ejemplo de un biopolímero antiguo, en cuanto que el quitosano es uno de los biopolímeros mas recientes (Azevedo et al., 2007).

Los materiales textiles han presentado un papel cada vez mas importante en el dominio médico, que abarca mas que el vestuário de protección médico. Las fibras desarrolladas, tejidos, telas y notejidos pueden ser aplicados en no-implantes, implantes y dispositivos extra-corporales (Araújo et al., 2000ab).

En este trabajo, los autores desarrollaron una fibra textil que fue producida a partir de polímeros con la celulosa y el quitosano provenientes del aprovechamiento de resíduos como el bagazo, paja y quitosano derivado de la quitina. La fibra híbrida producida une 
las propiedades y características de los dos polímeros, presentando en el núcleo la celulosa y en el revestimiento externo el quitosano con sus propiedades terapeuticas que viabilizan las aplicaciones médicas. En terminos de tenacidad las fibras obtenidas de la celulosa del bagazo y de la paja fueron compatibles con el Lyocell. Las fibras producidas serán probadas futuramente para la producción de estructuras textiles y membranas, con propiedades específicas para el tratamento de dermatitis.

El aumento de la expectativa de la vida del hombre, como consecuencia de los adelantos de la medicina tienen resultados en el aumento de la esperanza de vida y, por consecuencia, de las enfermedades relacionadas con la vejez. Entre estas enfermedades, se destacan las enfermedades dermatológicas, que interfieren profundamente en la vida de los indivíduos, ya sea por el comezón, por el aspecto no estética, o por restricciones impuestas por las lesiones y por el propio tratamiento (Alvarenga y Caldeira, 2009).

En lo que se refiere al desarrollo de vendajes para el tratamiento de lesiones de la piel, uno de los desafios es la adecuación del tipo de lesión (por ejemplo, aguda, crónica, exsudativas o seca) al curativo. De esta forma, ningun curativo es apropiado para el tratamiento de todos los tipos de lesiones. La propuesta de esta investigación esta relacionada con las cuestiones de innovaciones y en los benefícios que pueden ser ofrecidos a la sociedad.

\section{Materiales y métodos}

\subsection{Bagazo y la paja de caña de azúcar}

El bagazo y la paja de caña de azúcar utilizado en este trabajo fueron gentilmente cedidos por la Usina Azucarera Ester S.A, de la ciudad de Cosmópolis, en el Estado de São Paulo. El bagazo de la caña de azúcar en su forma bruta fue previamente caracterizado conteniendo $43,7 \%$ de glucano, 27,6\% de poliosis, 22,4\% de lignina Klason, $1 \%$ de lignina soluble en $\mathrm{H}_{2} \mathrm{SO}_{4}$ e 2,8\% de cenizas. La paja también fue caracterizada conteniendo $33,5 \%$ de glucano, $27,1 \%$ de poliosis, $21 \%$ de lignina Klason, $4,8 \%$ de lignina soluble en $\mathrm{H}_{2} \mathrm{SO}_{4}$ e $2,5 \%$ de cenizas

El bagazo fue secado al aire por cerca de 10 días hasta alcanzar un nivel de humedad de aproximadamente $10 \%$; en seguida fue embalado en sacos de polipropileno y guardado en cámara fría a $4^{\circ} \mathrm{C}$.

El tamaño promedio de las partículas de bagazo utilizado fue de 8 a $60 \mathrm{~mm}$ de largo y 0,4 $\mathrm{mm}$ de diámetro de un filamento único siendo $4 \mathrm{~mm}$ o diámetro de la aglomeración. Esas medidas fueron realizadas utilizando un calibrador Mitutoyo (0 a $250 \mathrm{~mm}$ ) disponible en el Departamento de Tecnología Bioquímico-Farmacéutica, Facultad de Ciencias Farmacéuticas, USP/SP. 


\subsection{Despulpados soda / AQ}

Las pulpas $\mathrm{NaOH} / \mathrm{AQ}$ fueron obtenidas utilizando $4,5 \mathrm{~g}$ de bagazo o paja de caña y solución de $\mathrm{NaOH} 20,64 \%(\mathrm{~m} / \mathrm{v})\left(16 \%\right.$ de $\mathrm{Na}_{2} \mathrm{O}$ activo) obedeciendo la relación licor: bagazo de $12: 1(\mathrm{v} / \mathrm{m})$ e $0,15 \%(\mathrm{~m} / \mathrm{v})$ de AQ en relación a la masa seca de bagazo. La reacción fue hecha en un reactor de acero inoxidable, con capacidad de $500 \mathrm{~mL}$, a $160^{\circ} \mathrm{C}$ por 120 min, disponible en el Laboratorio de Textiles y confecciones del Centro Tecnológico de la Industria de la Moda del Instituto de Investigaciones Tecnológicas-IPT. El tiempo de calentamiento hasta la temperatura de $160^{\circ} \mathrm{C}$ previamente determinado fue de $90 \mathrm{~min}$, resultando en un tiempo to0tal de proceso de $210 \mathrm{~min}$. Las pulpas obtenidas fueron exhaustivamente lavadas con agua destilada hasta alcanzar $\mathrm{pH} 7$ y fueron secadas a $25^{\circ} \mathrm{C}$. Para obtener $100 \mathrm{~g}$ de pulpa fueron realizadas cerca de 70 reacciones.

Los rendimientos de la spulpas fueron calculados por la ecuación (la masa seca se refiere al material sin humedad)

$$
\mathrm{RT}=\frac{m}{M} \times 100 \quad(\text { ecuación } 1)
$$

En que:

$\mathrm{RT}=$ Rendimiento total $(\%)$;

$\mathrm{M}=$ masa de bagazo (g) (base seca);

$\mathrm{m}=$ masa de pulpa obtenida trás el final del despulpado $(\mathrm{g})$ (base seca).

\subsection{Clasificaciones de las pulpas de bagazo y paja}

Para la clasificación de las pulpas de bagazo y paja de caña, cada muestra fue desagregada (consistencia aproximadamente 0,3\%) en aparato MA-1032. La suspensión de pulpa fue adicionada en un clasificador del tipo Somerville con ranura de $0,15 \mathrm{~mm}$. Las pulpas clasificadas fueron centrifugadas y guardadas en cámara fría a $4^{\circ} \mathrm{C}$.

\subsection{Blanqueamiento de las pulpas}

En cada muestra de pulpa clasificada, inicialmente fue realizada una secuencia de blanqueamiento químico constituído por três etapas: extracción alcalina con hidróxido de sodio (E), tratamiento de quelación com DPA ácido dietileno triamina penta-acético $(\mathrm{Q})$ y tratamiento con peróxido de hidrógeno $(\mathrm{P})$ usando las mejores condiciones encontradas por la FARIA (1994). 


\subsection{Preparación de las fibras textiles}

\subsubsection{Fibras de celulosa de bagazo y paja}

Las fibras fueron preparadas utilizando $10 \%$ de celulosa, adicionando $80 \%$ de óxido de $\mathrm{N}$ metilmorfolina (NMMO) y $10 \%$ de agua a $75^{\circ} \mathrm{C}$ por $30 \mathrm{~min}$. La extrusión de las fibras fue hecha en un baño conteniendo agua a temperatura ambiente conforme a la metodología (Fink et al., 2001).

\subsubsection{Fibras de Quitosano}

Las fibras fueron preparadas disolviéndose el quitosano 2,5\% $(\mathrm{m} / \mathrm{v})$ en una solución de ácido acético $2 \%(\mathrm{~m} / \mathrm{v})$. Tras la preparación del gel las fibras fueron extruidas en una solución de precipitación conteniendo hidróxido de sodio $1 \mathrm{M}$ (Tzulakoglu et al, 2005).

\subsubsection{Fibras híbridas de celulosa/quitosano}

Las fibras fueron preparadas disolviéndose $10 \%(\mathrm{~m} / \mathrm{m})$ de celulosa en $80 \%(\mathrm{~m} / \mathrm{m})$ de óxido de N-metilmoorfolina (NMO) y $10 \%(\mathrm{~m} / \mathrm{m})$ de agua. La mezcla fue colocada en baño maría a $75^{\circ} \mathrm{C}$ durante un período de $40 \mathrm{~min}$ (Fink, et al, 2001). La extrusión del gel obtenido fue hecha en una solución de quitosano $0,5 \%(\mathrm{~m} / \mathrm{v})$ disuelta en ácido acético $2 \%(\mathrm{~m} / \mathrm{v})$. Las fibras de celulosa fueron mantenidas en la solución de quitosano por $24 \mathrm{~h}$. Luego de este período de tiempo las fibras fueron lavadas con agua desionizada y envueltas en conos de polipropileno. Las fibras fueron secadas a temperatura ambiente.

\section{Resultados y discusión}

\subsection{Fibras textiles obtenidas}

La figura 2 muestra el aspecto de las fibras textiles a) celulosa bagazo, b) celulosa paja y c) celulosa/quitosano. Se puede observar que las fibras están orientas en una dirección y presentan cierta regularidad a pesar del proceso de extrusión manual. 


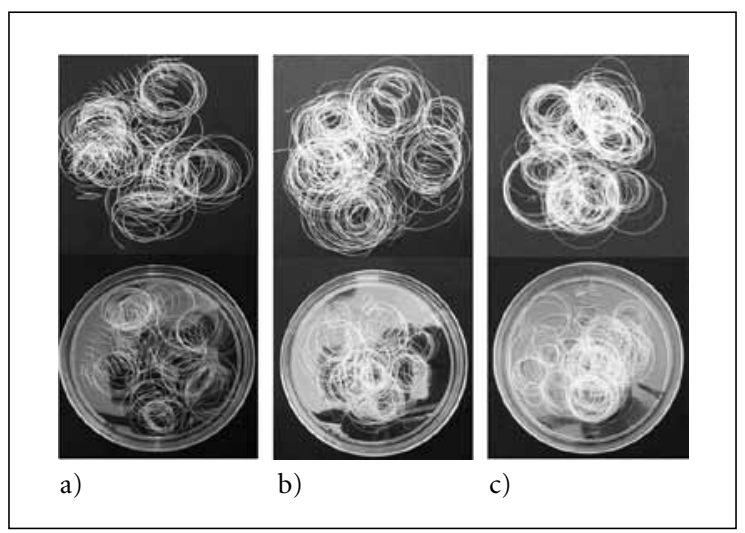

Figura 2. Aspecto de las fibras textiles a) celulosa bagazo, b) celulosa paja y c) celulosa/quitosano. El diámetro de cada placa corresponde a $10 \mathrm{~cm}$.

\subsection{Caracterización de las fibras}

Se puede observar en la Tabla 1 que las fibras obtenidas de la celulosa de bagazo y paja fueron las que presentaron valores de tenacidad de $4,3 \mathrm{cN} /$ tex, compatibles con las fibras de liocel según los datos de la literatura 4,24-4,41 cN/tex (Maluf y Kolbe, 2003).

\begin{tabular}{|l|c|c|c|}
\hline Muestra de fibras & $\begin{array}{c}\text { Carga de ruptura } \\
(\mathrm{N})\end{array}$ & $\begin{array}{c}\text { Título } \\
(\text { Tex })\end{array}$ & $\begin{array}{c}\text { Tenacidad } \\
(\mathrm{Cn} / \mathrm{tex})\end{array}$ \\
\hline Celulosa bagazo (A) & $1,1 \pm 0,45$ & 25,7 & 4,3 \\
Celulosa paja (B) & $1,1 \pm 0,68$ & 26,2 & 4,2 \\
Quitosano & $1,17 \pm 0,20$ & 29,9 & 3,9 \\
Híbrida A/ Quitosano & $1,13 \pm 0,15$ & 33,9 & 3,3 \\
Híbrida B/ Quitosano & $1,13 \pm 0,15$ & 33,9 & 3,3 \\
\hline
\end{tabular}

Tabla 1. Tenacidad de fibras acondicionadas $\left(20^{\circ} \mathrm{C} / 65 \%\right.$ U.R. $)$ Fuente. Elaboración propia.

La resistencia a la tracción va a depender Del tipo de fibra, Del proceso de producción (fibras manufacturadas de tenacidad normal, media o elevada), de la espesura (o diámetro) y de la distancia entre garras del dinamómetro. Cuanto menos sea la distancia inicial entre las garras del dinamómetro mayor será la resistencia de la fibra o tejido (Maluf y Kolbe, 2003). En vista de todas las variables que influyen en la resistencia a la tracción, tomándose una 
pequeña muestra de fibras con espesuras diferentes, es probable que sean encontradas diferentes cargas de ruptura. Por este motivo, para tenerse una idea de la resistencia de una fibra, se determina su carga de ruptura y divide por el título de la fibra (Maluf y Kolbe, 2003). Variables tales como la elongación son importantes, por ejemplo, en el caso de las compresas aplicadas para el tratamiento de heridas. Las compresas son utilizadas en múltiples aplicaciones, incluyendo las infecciones, la absorción y exudación de sangre y de fluidos, cicatrización y aplicaciones de medicamentos. Idealmente, las compresas quirúrgicas deben ser suaves, flexibles, cubrir la herida con el fin de protegerla de la contaminación, que sean fáciles de poner y quitar, que sean estériles y no presenten toxicidad (Araujo et al, 2001a).

\section{Conclusiones}

En este trabajo, la atención fue puesta en el proceso de extracción de celulosa bagazo e de la paja de caña y quitosano, preparación y la propiedad de las fibras para uso potencial en aplicaciones textiles. Los ensayos mecánicos mostraron que tanto las fibras de la celulosa y de la paja tienen resistencia a la tracción compatible con fibras de liocel encontrado en el mercado textil.

\section{Gracias}

Los autores agradecen el apoyo financiero de la Fundação de Amparo à Pesquisa do Estado de São Paulo - FAPESP.

\section{Referencias Bibliográficas}

Alonso, D., Gimeno, M., Sánches, S. J. D.; Shirai, K. (2010). Chitosan-based microcapsules containing grapefruit seed extract onto cellulose fibers by a non-toxic procedure. Carbohydrate Research, v. 345, p. 854-859.

Alvarenga, T. M. M.; Caldeira, A. P. (2009). Qualidade de vida em pacientes pediátricos com dermatite atópica. Jornal de Pediatria, v. 85, n.5, p. 415-420.

Araújo, M., Fangueiro, R., Hong, H. (2001a). Têxteis Técnicos: Materiais do Novo Milénio, Ed. Williams, Lda, Ministério da Economia, v. 2, p. 167.

(2001b). Têxteis Técnicos: Materiais do Novo Milénio, Ed. Williams, Lda, Ministério da Economia, v.3, p. 187.

Azevedo, V. V. C.; Chaves, S. A.; Bezerra, D.C.; Lia Fook M. V.; Costa, A. C. M. (2007). Qui- 
tina e Quitosana: aplicações como biomateriais. Resvista eletrônica de Materiais e Processos v. 2.3.

Canella, K. M. C., Garcia, R. B. (2001). Caracterização de quitosana por cromatografia de permeação em gel - influência do método de preparação e do solvente. Química Nova, v. 24, p.13-17.

CONAB Companhia Nacional de Abastecimento, Produção nacional de grãos sobe para 157,8 milhões de toneladas. Disponible en www.conab.gov.br. Consultado el 10. 03.12.

Costa, S. M., Mazzola, P. G., Silva, J. C. A. R., Pahl, R., Pessoa, A., Costa, S. A. (2013). Use of sugar cane straw as a source of cellulose for textile fiber production. Industrial Crops and Products, v. 42, p. 189-194.

Costa, S. M., Costa, S. A., Pahl, R., Mazzola, P. G., Marcicano, J. P. P., Pessoa A. (2013)Textile fiber produced from sugarcane bagasse cellulose: an agro-industrial residue. International Journal of Textile and Fashion Technology (IJTFT), v. 2, pp. 15-28.

Costa, S. A., Pessoa, J. R. A., Costa, S. M. (2008). Fibra obtida a partir de resíduos agroindustriais, processo para sua preparação e produto compreendido para tal fibra, em 06.04.10, foi publicado na Revista da Propriedade Industrial - RPI No. 2048, p. 46.

Faria, L. F. F. (1994). Preparação de nitrato de celulose a partir de bagaço de cana de açúcar. FAENQUIL/Departamento de materiais, (Dissertação de mestrado).

Fengel, D., Wegener, G. (1989). Wood: Chemistry, Ultrastructure, Reactions. Berlin: Walter de Gruyter., pp. 133-145.

Ferraz, A. Aplicações da biotecnologia na produção de papel e celulose. En: Lima, U.A., Aquarone, E., Borzani, W., Schmidell, W. (ed) (2001). Biotecnologia Industrial. São Paulo: Edgard Blucher Ltda, .v. 3 pp. 465-484.

Fink, H. P., Weigel, P., Purz, H. J., Ganster, J. (2001). Structure formation of regenerated cellulose materials from NMMO-solutions. Progress in Poymer Science, v. 26, p. 1473-1524. Hoven, V. P., Tangpasuthadol, V., Angkitpaiboon, Y., Vallapa, N., Kiatkamjornwong, S. (2007). Surface-charged chitosan: Preparation and protein adsorption. Carbohydrate Polymers, v. 68, n. 1, p. 44-53.

Jayakumar, J., Prabaharan, M., Sudheesh Kumar, P. T., Nair, S. V., Tamura, H. (2011). Biomaterials based on chitin and chitosan in wound dressing applications. Biotechnology advanvances, v. 29, n.3, pp. 322-337.

Kong, M., Chen, X. G., Xing, K., Park, H. J. (2010). Antimicrobial properties of chitosan and mode of action: A state of the art review. International Journal of Food Microbiology, v. 144, pp. 51-63.

Machado, G. O. (2000). Preparação e caracterização CMC e CMC graftizada. São Carlos, Instituto de Química de São Carlos, Universidade de São Paulo, p. 101. (Dissertação de mestrado).

Maluf, E., Kolbe, W. (2003). Fibras têxteis. Dados técnicos para a Indústria têxtil, Associação Brasileira da Indústria têxtil e de confecção ABIT. Instituto de Pesquisas tecnológicas, p. 25.

Pandey, A., Soccol, C. R., Nigan, P., Soccol, V.T. (2000). Biotechnology potential of agro-in dustrial residues. I: sugarcane bagasse. Bioresource Technology, v. 74, pp. 69-80.

Pillai, C. K. S., Paul, W., Sharma, C. P. (2009). Chitin and chitosan polymers: Chemistry, solubility and fiber formation, Progress in Polymer Science, v. 34, pp. 641-678. 
Programa de Agricultura e Meio Ambiente WWF -BRASIL. (2008) Análise da expansão do complexo agroindustrial Canavieiro no brasil. Disponible en: wwww.homologa.ambiente.sp.gov.br/etanolverde/.../rel_cana_wwf.pdf. Consultado el 05-03-2012.

Rosenau, T., Hofinger, A., Potthast, A., Kosma, P. (2003). On the conformation of the cellulose solvent N-methylmorpholine-N-oxide (NMMO) in solution. Polymer, v. 44, n. 2 , p. $8177,8182$.

UNICA - União da Indústria de Cana-de-Açúcar. Safra de cana de açucar 2008/09. Projeções apontam para crescimento de $16 \%$ no volume de cana colhida, com 32 novas usinas entrando em operação na região Centro-Sul ao longo da safra. Disponible en: www.portaldoagronegocio.com.br/conteudo.php?id=21696. Consultado el 05 07-2008.

Tolaimate, D. J., Rhazi, M., Alaguí, A., Vicendon, M., Vottero, P. (2000). On the influence of deacetylation process on the physicochemical characteristics of chitosan from squid chitin. Polymer, v. 41, p. 2463-2469.

Triana, O., Leonard, M., Saavedra, F., Fernádez, N., Gálvez, G., Peña, E. (1990). Atlas del bagazo de la cana, Cuba 9. Geplacea. Pnud. Icididca.

Tuzlakoglu, K., Alves, C.M., Mano, J.F., Reis, R.L. Production and characterization of chitosan fibers and 3-D fiber mesh scaffolds for tissue engineering applications.

Summary: Agribusiness in Brazil always figured prominently in the economy and the country's development. Currently, most of the residues of sugar cane burned in energy power stations. These residues are mainly composed of cellulose, lignin and polyoses. In this paper these compounds were used to produce textile fibers. To remove the cellulose was carried out the alkaline pulping and bleaching with hydrogen peroxide. The fibers obtained were consistent with Lyocell.

Keywords: cellulose - fabric - Lyocell - residues - straw - sugar cane.

Resumo: Os agronegócios no Brasil ocuparam sempre um sítio de destaque na economia y o desenvolvimento do país. Os resíduos agroindustriais como o bagaço e a palha da cana de açúcar são utilizados como matérias prima de fibras textiles.

Palavras chave: bagaço de cana de açúcar - celulosa - fibras palha - Lyocell - têxteis. 